\title{
THE FUNDAMENTAL SIGNIFICANCE OF THE SOCIAL AND ETHICAL CRITERIA FOR PRIMARY CARE QUALITY ASSESSMENT
}

\author{
B. Vasileva, Al. Vodenicharova \\ Faculty of Public Health \\ Medical University - Sofia, Bulgaria
}

\begin{abstract}
A vital aspect of the medical activity on which the medical care quality directly depends has been the doctor-patient relationship; it is of complex nature and is formed by a number of factors: professional ethics, communication culture of the doctor, the patients' confidence in the doctor, etc. From an ethical point of view, the confidence of the patient has been an ethical indicator and criterion of particular importance for the quality and interpersonal relationship between the doctor and the patient. Data from the overview of a number of papers, as well as data from our own empirical study, has indicated the presence of a number of unresolved problems in this relationship. That has raised the significant necessity of a more thorough and comprehensive training of the medical staff in professional medical ethics in undergraduate and postgraduate training.
\end{abstract}

Key words: ethical criteria, priorities, doctor-patient relationship, confidence, sociological information

Corresponding author: Assoc. Prof. A. Vodenicharova, Faculty of Public Health, Medical University, 8 Byalo More Str, University Hospital "Tsaritsa Yoanna", 1527 Sofia, e-mail: a.vodenicharova@foz.mu-sofia.bg

\section{INTRODUCTION}

M edical activity has been a type of specific interpersonal and social relationships. The patient-doctor relationships and contacts could be analyzed ethically as to how well the goals of medicine were achieved without harming the patient, the doctor-patient cooperation, the patient autonomy, fairness, and many other values and ethical issues.

There had been different approaches to the ethical and legal regulation of the doctor-patient relationships. From an ethical aspect, the generally competing two main approaches to the doctor-patient relationship had been of particular importance:

- autonomy;

- paternalism.
Autonomy came from the idea that the patient was not a passive object of medical impact, but an autonomous person with his or her own culture, awareness, opinions and values. Nowadays, the patient autonomy had been much more pronounced than in the past. According to international human rights instruments, a person has the right to life, health, liberty and security, as well as the right to self-determination. The complexity of this connection - between patient rights and human rights could be illustrated by a number of examples.

Paternalism as a model of doctor-patient relationships had a huge impact on the entire history of medicine, on the way of thinking and the approach to treatment and healthcare.

Paternalism was based on the idea that the physician had a better understanding of the patient's interests compared 
to the patient, and that was related to the specificity of his or her knowledge, as well as to the limitations caused by the sick patient experience. In addition, the professional responsibilities of the physician suggested that he or she would do all necessary and useful things for health even when the patient did not consider it to be good.

It should be emphasized that both autonomy and paternalism must not be absolutized in the process of activity and the doctor-patient relationship. Today, these two approaches had been largely synthesized, combined into one, as effective as possible - partnership as the main mechanism of interaction between doctor and patient throughout the diagnostic and treatment process.

\section{PUBLICATIONS AND ANALYSES ON ETHICAL ASPECTS OF MEDICAL CARES}

There had been a lot of literary sources on the ethical issues in medicine. Here it would be highlighted some of them related to the topic of this paper. M. Gardeva had made an interesting analysis of the ethical perspectives in assessing the needs of health services. She had emphasized that a fundamental principle of medical ethics was that health care decisions should lead to the greatest benefit for the individual patient, and that the main consideration should be to enhance individual health and safety. As stated in the fundamental moral right of the philosopher Immanuel Kant, we should not approach the individual with other intentions except solely as stimulating his or her development and realization [4].

The communicative culture played a decisive role in adequate doctor-patient relationships as part of the physician's professionalism. Bulgarian authors had paid special attention to the "listening" skill to the patient's story that was mainly the skill to take down the patient's medical history (R. Zlatanova, T. Zlatanova, B. Borisova, etc.). Data from publications by these authors had shown that, according to $43 \%$ of the patients, their GP always listened to them carefully, while $11 \%$ believed that the doctor rarely or never listened to them with attention, which diminished the patients' confidence in the doctor [5].

According to $\mathrm{B}$. Borisova, the general practitioner did not always want the opinion and consent of the patient on the various diagnostic and treatment procedures. In 44.3\%, the doctor rarely asked for the patient's opinion or did not want it at all. A total of $35.5 \%$ of doctors always asked for the patient's opinion, with a higher percentage for Sofia and a lower rate for the regional cities [1]. These data had revealed problems and gaps in the effective partnership between the doctor and the patient.

The author had emphasized that was an indicator of serious problems in the cooperation and communication be- tween GPs and the patient. The physician had imposed a strong pattern on the patient behavior and the patient had not been actively involved in the process of his or her own treatment; he or she did not have a sense of control over it. It was the well-described in literature paternalistic model where the physician had a strongly dominant role with the patient's passive position [1].

According to N. Shtereva-Nikolova, the good communication skills did not work well with most doctors; these skills should be developed and maintained with conscious efforts and periodic reviews [7].

\section{DATA FROM OUR OWN EMPIRICAL STUDY}

An anonymous sociological survey of 1053 patients was carried out regarding their relationship with the GP. Special attention was paid to the important ethical issue of patients' awareness of their rights. It was found that a great rate of respondents were poorly or not at all aware of the patients' rights. Only $20 \%$ of the respondents were well informed about the patients' rights while over half of them (52\%) were not informed at all.

It should be noted that patients' awareness of their rights was not only their personal problem, but also an organizational problem for the healthcare system itself and in particular of the general practitioners.
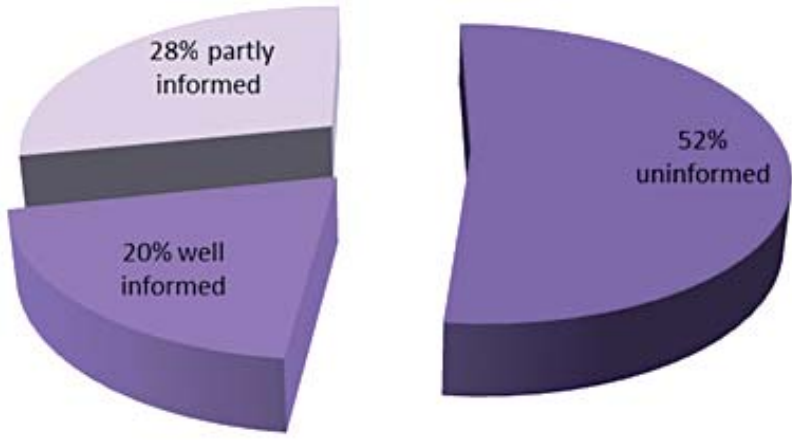

Fig. 1. Awareness of patients' rights

One of the leading and specific ethical criteria was the physician's responsiveness to the patient's problems and needs. That criterion had often been underestimated in the analyses however it was namely the patient's sense of the physician's responsiveness that had largely determined his or her satisfaction with the physician. Responsiveness was not only an ethical but also an organizational problem for medical care.

The data presented in Figure 2 showed quite clearly the unsatisfactory assessment of the patients surveyed about the responsiveness of their GPs. Over one-third of the surveyed patients $-39 \%$, considered the responsiveness to be low or missing, which was undoubtedly a serious ethical problem. Of course, the opinion on responsiveness was of subjective nature, but it had a significant influence on the patient's 


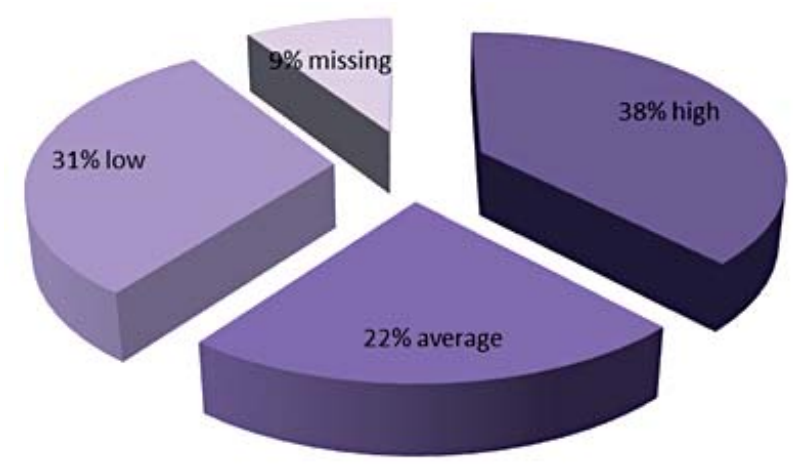

Question: How do you assess the doctor's responsiveness to your problems, questions and wishes?

Fig. 2. Assessment of the general practitioner's responsiveness

confidence in the doctor and in general on the doctorpatient relationship, and therefore it should be constantly analyzed. It should also be taken into account that the physician's responsiveness represented an important factor in the quality of health care.

Another important ethical and social and psychological problem in the doctor-patient relationship was the level of the patient's confidence in the doctor. Figure 3 showed that a significant ratio of the respondents had confidence in their GP. However it was impressive that $16 \%$ of them did not have confidence which was a problem in their relationships and an indicator of the patients' satisfaction.

There was a slight difference in the confidence in the physician among the surveyed rural and urban residents (Figure 2). The confidence of the rural residents was lower compared to the urban ones. The reason for that difference in the confidence in the general practitioner was of complex nature however the different qualification of the physicians in the rural and urban areas was important. The qualification of the GPs in the villages was, according to some observations, lower than the qualification of the GPs in the cities. Therefore, special analyses and approaches were required in this trend to systematically improve the qualifications of GPs working in the villages.

The doctor's skill to talk and "listen to the patient" was unsatisfactory. Almost half of the respondents (46\%) gave a definite negative answer.

The data also revealed the unsatisfactory assessment of the surveyed patients of their GPs responsiveness. Over one-third - 39\% thought that responsiveness was low or not shown at all.

\section{CONCLUSION}

The ethical evaluation of the medical activity had been determined first and foremost by the nature of the doc-

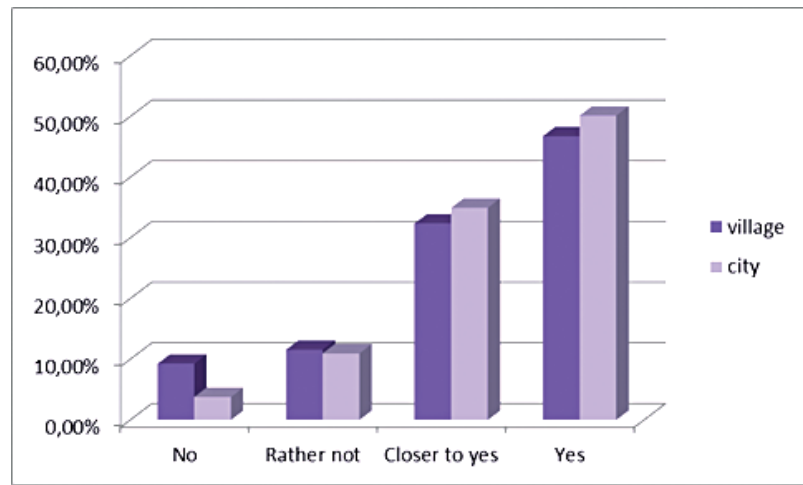

Question: Do you fully trust your GP for any and all health and personal issues?

Fig. 3. Patient confidence in the GP according to the place of residence $(\%)$

tor-patient relationship. That relationship had been a key factor and criterion for the quality of care provided.

The analyses of a number of authors, as well as our own empirical study, had shown the presence of certain unresolved problems in that relationship. The GPs communication culture had been insufficient.

The GPs communication skills should be recognized and regulated as important criteria for the overall assessment of his or her professionalism.

It should be appropriate that the issues of professional medical ethics, and in particular the communication culture of the general practitioners to be more broadly covered in the training of the medical students and in the curriculums of the faculties of public health.

Disclosure Summary: The authors have nothing to disclose.

\section{REFERENCES}

1. Borisova B. Evaluation of GP Activity through Patient Opinion. PhD Thesis, 2013, p. 162.

2. Valentinova Ts., Goranov M. The General Practitioners and the Evaluation of their Patients. General Practice, vol. V, 2, 2003, 3-5.

3. Vodenicharov Ts, Popova S, Medical Ethics, publ. house EkoPrint, Sofia, 2010.

4. Gardeva, M. Ethical problems in the assessment of health services needs and their prioritization. - Medical Meridians, 2013,3.

5. Zlatanova, T., R. Zlatanova-Velikova. Assessment of the Communication Skills of the GP. Jour. Medical Meridians, 2012, 1-2, 48-52.

6. Vasileva, B. Patient Satisfaction with GPs as a Social and Ethical Criterion for the Health Care Quality in the Pleven Region. PhD Thesis, 2019, 151.

7. Shtereva-Nikolova N. Communication and the Right to Information as a Major Component of Informed Consent. Social Medicine, 2015, 1.2: 29-31.

8. Beauchamp T, Childress J. Principles of Biomedical Ethics. Oxford university press, 2013.

9. Marshall M, Mannion R, Nelson E, Davies H.Managing change in the culture of general practice: qualitative case studies in primary care trusts. - BMJ, 2003; 327:599.

Received: October, 2019 - Accepted: October, 2019 